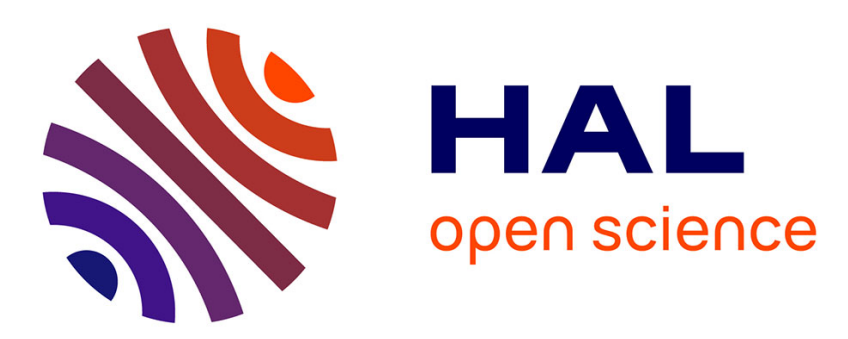

\title{
Partner selection for decode-and-forward cooperative relaying: A matching theoretic approach
}

\author{
Cengis Hasan, Eitan Altman, Jean-Marie Gorce
}

\section{To cite this version:}

Cengis Hasan, Eitan Altman, Jean-Marie Gorce. Partner selection for decode-and-forward cooperative relaying: A matching theoretic approach. IEEE PIMRC - 24th International Symposium on Personal Indoor and Mobile Radio Communications, Sep 2013, London, United Kingdom. pp.22752280, 10.1109/PIMRC.2013.6666523 . hal-00926587

\section{HAL Id: hal-00926587 https://hal.inria.fr/hal-00926587}

Submitted on 9 Jan 2014

HAL is a multi-disciplinary open access archive for the deposit and dissemination of scientific research documents, whether they are published or not. The documents may come from teaching and research institutions in France or abroad, or from public or private research centers.
L'archive ouverte pluridisciplinaire HAL, est destinée au dépôt et à la diffusion de documents scientifiques de niveau recherche, publiés ou non, émanant des établissements d'enseignement et de recherche français ou étrangers, des laboratoires publics ou privés. 


\title{
Partner Selection for Decode-and-forward Cooperative Relaying: A Matching Theoretic Approach
}

\author{
Cengis Hasan ${ }^{\dagger}$, Eitan Altman ${ }^{\ddagger}$, and Jean-Marie Gorce ${ }^{\dagger}$ \\ ${ }^{\dagger}$ Inria, University of Lyon, INSA-Lyon, 6 Avenue des Arts 69621 Villeurbanne Cedex, France \\ ${ }^{\ddagger}$ Inria, 2004 Route des Lucioles, 06902 Sophia-Antipolis Cedex, France \\ \{cengis.hasan, eitan.altman\}@inria.fr, jean-marie.gorce@insa-lyon.fr
}

\begin{abstract}
A matching theoretic approach to study the partner selection in cooperative relaying is followed. Partner selection is considered as a special stable roommate problem where each player ranks its partners by some criterion. Each agent aims here at finding a "good" partner in order to exploit efficiently the spatial diversity achieved with cooperation. We adapt Irving's algorithm [3] for determining the partners of each player. The ranking criterion here is chosen to be outage probability such that each player comprises its own preference list according to outage probability from the lowest to the highest. The first player in the preference list provides the lowest outage probability. We introduce a decentralized version of Irving's algorithm. Then, we compare the results obtained by stable-matching with the global optimum and random selection results. From the computational results, we observe that stable-matching results are near to global optimum as well as superior than random selection in terms of average outage probability.
\end{abstract}

\section{INTRODUCTION}

Let be considered a scenario where a set of users, or agents, aim at transmitting their own data to a common destination. This scenario may correspond for instance either to mobiles in a single cell in uplink mode or to a wireless sensor network with a single sink. The reference protocol used to transmit the whole information to the common destination relies on a layer 2 MAC approach which divides the resources and schedule the allocation of each resource to each agent. Even if the agents are all in the range of the destination, cooperative transmissions can significantly improve the efficiency of the system. The efficiency can be measured by the total capacity, the total energy or by any criteria related to the QoS such as the packet error rate. In this paper we focus on the outage probability. Transmission powers and capacity needs for each node are constant and cooperative transmission is used to reduce the outage probability. The formulation of the problem is based on the relay channel model as described in the early work of Laneman [1]. In the simplest approach, each source agent requires the help of another agent to improve its transmission by forming togeter an equivalent relay channel. We consider the special case where the agents associate by pairs such that each agent relays the data of the other. In the framework of network information theory, these nodes form a cooperative multiple access channel (CMAC).

This work is supported by the ANR project ECOSCells. It was done within the Inria-Alcatel Lucent Bell-Labs joint research laboratory.
Albeit the whole network can be considered as a large size CMAC, we rather propose to form several small coalitions. In this paper, these small coalitions are even limited to pairs. In order to optimize the overall performance of the network, the partner selection process is therefore crucial. Each agent aims here at finding a "good" partner in order to exploit efficiently the spatial diversity achieved with cooperation. This process can be identified as a matching problem. In the game theoretic sense where the players are "strategic decision makers", the partner selection process appears to be an example of the stable roommates problem. Stable matching theory was established by Shapley and Gale by their seminal work [2]. Gale and Shapley analyzed matching at an abstract, general level. They used marriage as one of their illustrative examples. How should ten women and ten men be matched, while respecting their individual preferences? The main challenge involved designing a simple mechanism that would lead to a stable matching, where no couples would break up and form new matches which would make them better off. The solution-the Gale-Shapley "deferred acceptance" algorithm-was a set of simple rules that always led straight to a stable matching.

The stable marriage problem is an example of a so called two-sided market due to the gender issue. However, this kind of problem can be broaden to matching problems with no gender issue and are referred to as one-sided market. This is the case of the stable roommates problem. In this problem, each person targets matching with the best partner to share a room. We shall show that the partner selection problem in the context of cooperative relaying can be studied as a onesided market. By determining the ranking rule of partners, we seek a stable matching. Although a stable matching is always possible in stable marriage problems, this is not the case in stable roommates problem. Further, even if a stable matching exists, there was no polynomial-time algorithm to find it until the recent work of Irving [3].

\section{A. Related Work}

The partner selection problem in cooperative communications has been already studied in the literature in [5] and [6]. More recently, Lee and Lee [7] extended the problem to relay assignment for multi-user DF-AF cooperative wireless networks while in [8] the authors proposed a new selection method which requires neither error detection methods at relay nodes nor feedback information at the source. The 
thesis in [9] includes many new approaches for matching the cooperating agents. In [10] the authors study the relay selection in heterogeneous relay networks, i.e. where relays with different protocols can co-exist. While varying algorithms are proposed in these papers, none of these paper uses the coalition formation principle. Cooperative game theoretic approaches exist in the literature for wireless problems, where "coalitions formation" problems are studied. A coalition can be of any size, from a single player to all players. For instance, [11] studies coalition formation of mobiles and destinations. Another coalitional game approach is formulated in [12] to examine how coalitions can form in a distributed manner, as well as possible resource allocation methods within groups. Moreover, in [13], a Markov chain model is proposed to investigate the stability of the coalitional structures.

\section{B. Our Contribution}

In this paper, we propose the use of matching theory and more specifically the stable roommates problem, to solve the partner selection problem in cooperative transmissions. This work shows that this formalism is perfectly convenient and further a natural tool for this problem. The reason is that it provides a fair and stable sectioning process if we consider the source nodes (agents) as strategic decision makers. Here, we use this tool to analyse the partner selection problem.

\section{Outline}

We introduce the mathematical background of stable matching games and stable roommates problem in Section II. The problem formulation and settings are given in Section III. In Section $\mathrm{V}$, the computational results are obtained and discussed.

\section{Stable Matching Games}

A matching game can be considered as a special NTU-game where the cardinality of each basic coalition is at most 2 so that $S=(i, j), i, j \in N$. Note that $i$ can be equal to $j$ which means a player is possible to be alone in coalition $S$. The stable marriage problem is primal problem formalised in the context of two-sided market [2].

Specifically, a matching $\mathcal{M}$ is a partition of the set of players $N$, which we denote as $\mathcal{M}(N)$ such that $\bigcup_{S \in \mathcal{M}(N)}=N$. The utility of player $i \in S$ is represented by $u_{i}^{S}$. Player $i$ might prefer to or be indifferent between coalition $S$ and $T$. The preference relation $\succeq$ is a reflexive, complete and transitive binary relation. $S \succ_{i} T \Leftrightarrow u_{i}^{S}>u_{i}^{T}$ means that player $i$ strictly prefers coalition $S$ to $T$.

Definition 2.1: A coalition $B$ is said to block a matching $\mathcal{M}$ whenever both $i \in S$ and $j \in T$ prefers coalition $B$ to $S$ and $T$, respectively, i.e. $B \succ_{i} S$ and $B \succ_{j} T$.

Definition 2.2: A matching $\mathcal{M}$ is said to be stable whenever there does not exist a blocking coalition.

\section{A. Stable Roommates Problem}

The stable roommates problem (SRP) corresponds to a onesided market. Each person aims to find his best roommate. Therefore, the preference list of a specific person is composed of a descending order all possible partners. Note that a player can also rank himself in the list. In this case, the player remains alone. Incomplete lists in a SRP mean that a person does not include all the roommates in his preference list.

The problem of finding stable bipartite coalitions of mobiles where a mobile is let to order his possible partners according to some preference relation can be seen a SRP as described in Section III.

\section{B. An Efficient Algorithm for Solving SRP}

The stable roommates problem had been a nontrivial open problem, until Irving [3] constructed the first polynomial time algorithm which determines whether a given instance of the stable roommates problem admits a stable matching, and if so, finds one [14]. Irving, in his paper [3], proves that the proposed algorithm has $O\left(n^{2}\right)$ complexity.

We name the algorithm here as alg-IRVING consisting of two phases:

\section{First Phase}

Each player in his turn do a bid to his partners. This sequence of bids proceeds with each individual pursuing the following strategies:

1) If $i$ receives a bid from $j$, then

a) he rejects it if he already holds ${ }^{1}$ a better bid from someone higher than $j$ in his preference list;

b) he holds it for consideration simultaneously rejecting his current bid being poorer than $j$.

2) If $i$ is rejected by someone in his preference list, he continues proposing until accepted by a partner.

This phase of algorithm will terminate

(i) with every person holding a bid, or

(ii) with one person rejected by everyone

In case of (ii), the algorithm will terminate with no matching meaning that the problem is not stable.

If the first phase of the algorithm terminates with every person holding a bid, then the preference list of possible partners for $j$, who holds a bid from $i$, can be "reduced" by deleting from it

\section{Reductions}

- all those to whom $j$ prefers $i$;

- all those who hold a bid from a person whom they prefer to $j$.

We denote as Reductions the procedure that performs these operations. If the reduced preference list of player $i$ and $j$ contains only $j$ and $i$, respectively, then it is said that they are 
matched; therefore, in the second phase of algorithm, they are out of consideration.

We denote by $\mathcal{S}$ the ordered pairs of the form $(x, y)$, where $y$ holds a bid from $x$. It is said that $y$ is $x$ 's current favorite which is the first in his reduced preference list.

\section{Second Phase}

The second phase of alg-IRVING deals with finding a rotation $\rho$ in $\mathcal{S}$. In case of a rotation, the set $\mathcal{S}$ is repeatedly changed by the application of rotations. After applying rotation, if two players are matched, then they are removed from $\mathcal{S}$.

A rotation relative to $\mathcal{S}$ is a sequence

$$
\rho(\mathcal{S})=\left\{\left(x_{1}, y_{1}\right),\left(x_{2}, y_{2}\right), \ldots,\left(x_{k}, y_{k}\right),\left(x_{1}, y_{1}\right)\right\}
$$

such that $\forall\left(x_{l}, y_{l}\right) \in \rho(\mathcal{S}), y_{l+1}$ is $x_{l}$ 's current second favorite in his reduced preference list.

If an even length rotation is found such that $x_{l+1}=y_{l}$ forall $l$, this is the case referred to even party which is also an indicator of no stable matching.

In case of no an even party, the application of rotation involves replacing the pairs $\left(x_{l}, y_{l}\right)$ in $\mathcal{S}$ by the pairs $\left(x_{l}, y_{l+1}\right)$ and performing again the procedure Reductions on the preference lists of corresponding players. The second phase continues until not finding a rotation which indicates that a stable matching is found.

Examples can bound in [3] and [4].

\section{Problem Formulation and Settings}

The system model is depicted in Fig.1. Let $N=(1, \ldots, n)$ be the set of players and $d$ the destination node, or base station (BS). In this paper we rather target the case where the players are in a common area, sufficiently far from the BS such that the inter-mobile channels are statistically better than the mobiledestination channels. This is a favourable situation for mobile cooperation. This assumption is not always necessary and most of our results apply for any scenario. However, especially in the CMAC case, this assumption may drive our settings.

In the default setting a resource unit (RU) is allocated to each player. These blocks may be time slots, frequency channels or time/frequency RU in LTE. We assume a perfect orthogonality between the blocks. Without loss of generality and for the sake of simplicity, we consider that each player receives a unique RU, and all have the same capacity.

\section{A. Cooperative Relaying}

Albeit it cannot achieve the upper bound capacity, the decode-and-forward strategy is nearly capacity achieving when the source-relay channel is much better than the others which is the case in our scenario here. We assume that each node is equipped only with one antenna. Interference-free uplink is considered where the transmissions of bipartite coalitions (in the sequel, we introduce these coalitions) do not interfere each other. Half-duplex transmission mode is applied in the communication between mobiles. There are adequate reasons for limiting the communication in half-duplex mode; because of insufficient electrical isolation between the transmit and receive circuitry, a terminal's transmitted signal drowns out the signals of other terminals at its receiver input [1]. All channels are assumed to be subject to slow varying block fading.

The physical channel between node $i$ and $j$ has the following instantaneous signal to noise ratio (SNR): $\gamma_{i, j}=$ $\Gamma_{i, j}\left|h_{i, j}\right|^{2}$, where $\left|h_{i, j}\right|$ is the Rayleigh distributed fading coefficient with variance $\sigma_{i, j}^{2}$. Moreover, we assume that $\forall i, j \in N, \sigma_{i, j}=1$. The term $\Gamma_{i, j}$ is the average SNR and is modelled as following:

$$
\Gamma_{i, j}=\left(\frac{P}{N_{0}}\right) S_{i, j} d_{i, j}^{-\beta},
$$

where

- $P$ is transmission power which is equal for all mobiles.

- $S_{i, j}$ is a zero-mean log-normal shadowing component with standard deviation $\sigma_{S}$.

- $d_{i, j}$ is the distance between nodes $i$ and $j$ as well as $\beta$ is the path loss exponent.

The SNR in the transmitter part is $P / N_{0}$. Both the fading and shadowing components are i.i.d for each $\{i, j\}$ pair. The shadowing components are constant for a given network realization and are assumed to be reciprocal, $S_{i, j}=S_{j, i}$.

\section{B. The Protocol}

We consider the following decode-and-forward protocol:

$\mathrm{S}_{1}$ : Source sends its data to relay and destination.

$\mathbf{S}_{2}$ : Relay tries to decode. If relay succeeds, then source and relay resend the packet. If relay fails, source resends alone.

\section{$\mathbf{S}_{3}$ : Destination combines all copies of data.}

We assume here that the source and relay transmit simultaneously without phase synchronization during $\mathbf{S}_{2}$, and these transmissions do not interfere each other.

In case of repetition coding at the relay, the mutual information $(\mathrm{bps} / \mathrm{Hz}$ ) can be readily shown to be

$$
\mathcal{I}_{i}= \begin{cases}\frac{1}{2} \log \left(1+\gamma_{i, d}^{\prime}+\gamma_{i, d}^{\prime \prime}+\gamma_{j, d}\right) & \text { if } \frac{1}{2} \log \left(1+\gamma_{i, j}\right)>R_{i} \\ \frac{1}{2} \log \left(1+\gamma_{i, d}^{\prime}+\gamma_{i, d}^{\prime \prime}\right) & \text { otherwise. }\end{cases}
$$

Here, source is $i$, and relay is $j$. Relay can retransmit the data of source with rate $R_{i} . \gamma_{i, d}^{\prime}$ and $\gamma_{i, d}^{\prime \prime}$ are the instantaneous SNRs of source-destination transmissions in $\mathbf{S}_{\mathbf{1}}$ and $\mathbf{S}_{\mathbf{2}}$ defined in the protocol as well as $\gamma_{j, d}$ is the instantaneous SNR of relay-destination transmission in $\mathbf{S}_{\mathbf{2}} \cdot \gamma_{i, d}^{\prime}$ and $\gamma_{i, d}^{\prime \prime}$ are assumed independent.

Remark 3.1: In this paper, we focus on the "selective" decode-and-forward transmission where the relay station only decodes the data and retransmits it to the destination. One can improve the context of this work by applying the compressand-forward transmission as well as MIMO attributes to the 


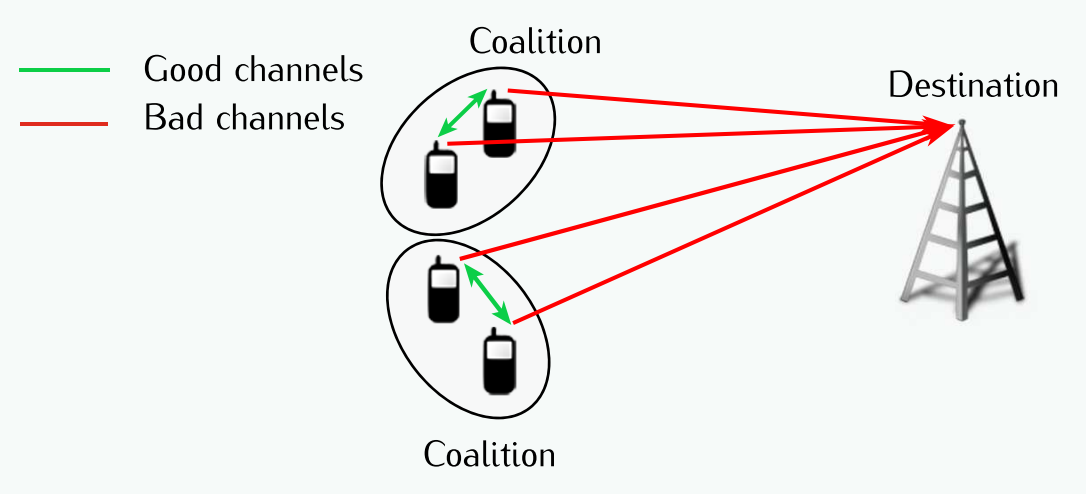

System model of the problem

Each mobile determines the partners according to the decentralized algorithm

The inter-mobile channels are assumed to be relatively much better than the mobile-destination channels

Fig. 1. The problem.

nodes. Moreover, the Rician fading channel model could be considered which might change significantly the couplings.

\section{Outage Probability Calculation}

1) Direct Transmission-No Cooperation: When a player $i$ stays alone the outage probability is given by [1]: $p_{\mathcal{O}}=$ $1-\exp \left(-\frac{2^{R_{i}}-1}{\sigma_{d}^{2} \Gamma_{i, d}}\right)$. This is a result when the player utilizes all degrees of freedom.

2) Cooperation: The outage probability can be calculated as following: $p_{\mathcal{O}}=p_{\mathcal{C}} p_{\mathcal{O} \mid \mathcal{C}}+\left(1-p_{\mathcal{C}}\right) p_{\mathcal{O} \mid \mathcal{N C}}$, where

- $p_{\mathcal{C}}$ is the probability of successfully reception of source's data at the relay given by

$$
\begin{aligned}
p_{\mathcal{C}} & =\operatorname{Pr}\left[\frac{1}{2} \log \left(1+\gamma_{i, j}\right)>R_{i}\right]=\operatorname{Pr}\left[\left|h_{i, j}\right|^{2}>\frac{2^{2 R_{i}}-1}{\Gamma_{i, j}}\right] \\
& =\exp \left(-\frac{2^{2 R_{i}}-1}{\sigma_{m}^{2} \Gamma_{i, j}}\right)
\end{aligned}
$$

Note that $|h|^{2}$ follows an exponential distribution.

- $p_{\mathcal{O} \mid \mathcal{C}}$ is the conditional probability of outage in destination when the relay decodes correctly the source's data:

$$
\begin{aligned}
p_{\mathcal{O} \mid \mathcal{C}} & =\operatorname{Pr}\left[\frac{1}{2} \log \left(1+\gamma_{i, d}^{\prime}+\gamma_{i, d}^{\prime \prime}+\gamma_{j, d}\right)<R_{i}\right] \\
& =\operatorname{Pr}\left[\gamma_{i, d}^{\prime}+\gamma_{i, d}^{\prime \prime}+\gamma_{j, d}<2^{2 R_{i}}-1\right],
\end{aligned}
$$

where $\gamma_{i, d}^{\prime}$ and $\gamma_{i, d}^{\prime \prime}$ are independent. The sum of $k$ exponential random variables $X=\sum_{i=1}^{k} X_{i}$ where each $X_{i}$ has different mean $\lambda_{i}$, follows hypo-exponential distribution of which the probability density function is given by

$$
f_{X}(x)=\sum_{i=1}^{k} \lambda_{i}\left(\prod_{j=1, j \neq i}^{k} \frac{\lambda_{j}}{\lambda_{j}-\lambda_{i}}\right) \exp \left(-\lambda_{i} x\right) .
$$

The sum $\gamma_{i, d}^{\prime}+\gamma_{i, d}^{\prime \prime}+\gamma_{j, d}$ follows a 3rd order hypoexponential distribution with means $\lambda_{1}=\lambda_{2}=$

$$
\begin{aligned}
& \left(\Gamma_{i, d} \sigma_{d}^{2}\right)^{-1} \text { and } \lambda_{3}=\left(\Gamma_{j, d} \sigma_{d}^{2}\right)^{-1} \text {. Therefore, } \\
& p_{\mathcal{O} \mid \mathcal{C}}=1-\frac{e^{-\frac{2^{2 R-1}}{\sigma_{d}^{2} \Gamma_{i, d}}} \Gamma_{i, d}\left(\sigma_{d}^{2} \Gamma_{i, d}+2^{2 R}-1\right)}{\sigma_{d}^{2}\left(\Gamma_{i, d}-\Gamma_{j, d}\right)^{2}} \\
& +\frac{e^{-\frac{2^{2 R}-1}{\sigma_{d}^{2} \Gamma_{i, d}}} \Gamma_{j, d}\left(2 \sigma_{d}^{2} \Gamma_{i, d}+2^{2 R}-1\right)}{\sigma_{d}^{2}\left(\Gamma_{i, d}-\Gamma_{j, d}\right)^{2}}-\frac{\Gamma_{j, d}^{2} e^{-\frac{2^{2 R}-1}{\sigma_{d}^{2} \Gamma_{j, d}}}}{\left(\Gamma_{i, d}-\Gamma_{j, d}\right)^{2}} .
\end{aligned}
$$

- $p_{\mathcal{O} \mid \mathcal{N C}}$ is the conditional outage probability when relay fails and source repeats its own data:

$$
\begin{aligned}
p_{\mathcal{O} \mid \mathcal{N C}} & =\operatorname{Pr}\left[\frac{1}{2} \log \left(1+\gamma_{i, d}^{\prime}+\gamma_{i, d}^{\prime \prime}\right)<R_{i}\right] \\
& =\operatorname{Pr}\left[\gamma_{i, d}^{\prime}+\gamma_{i, d}^{\prime \prime}<2^{2 R_{i}}-1\right] \\
& =1-\left(\frac{2^{2 R}-1}{\sigma_{d}^{2} \Gamma_{i, d}}+1\right) e^{-\frac{2^{2} R_{-1}}{\sigma_{d}^{2} \Gamma_{i, d}}}
\end{aligned}
$$

\section{Preference Functions}

Here, we intend to show how a mobile designs its preference list which is the ranking of possible partners (including itself) from the most preferable to the least.

Long Term-Outage Probability Ranking: The motivation here is to determine a long-term partnership. The channel state information has statistical characterization, for example, the type of fading distribution. We can utilize this characterization in determining the partnership. By knowing the variance of the fading each mobile is able to calculate the outage probability. Thus, a mobile evaluates its partners by means of that metric. The preference list of each mobile is composed of ranking the possible partners according to the outage probability in a way that

\section{the first ranked provides the lowest outage probabil- ity}

In that setting, each mobile also ranks itself in the preference list. 


\section{E. Decentralized Approach to the alg-IRVING}

We consider that each mobile is able to communicate in a separated control channel to look for partners.

\section{Learn:}

- Each mobile listens to the other partners continuously or randomly when each of them broadcast his averaged path loss and shadowing.

- Each mobile maintains a preference list from the messages sent by the other mobiles.

\section{Phase 1:}

- Randomly, each mobile does a bid until accepted by a partner in his preference list.

- Each mobile deletes some partners from his preference list according to Reductions procedure.

Phase 2:

- Each mobile broadcasts his second player of preference list to the other mobiles

- Each mobile performs rotation according to the received message. In case of an even party, then each mobile transmits alone without cooperation.

- Each mobile runs Reductions procedure according to the rotation, and continues Phase 2 until having only one partner in the preference list.

\section{Global Optimum}

In this section, we analyse the problem in terms of global optimum. The aim is to measure how much the stable matching is far from the global optimum.

\section{A. Minimum Total Outage Probability}

In terms of global outage minimization, the problem can be considered as a special case of the classical set-partitioning problem, which aims at finding the best partition of the $N$ players which minimizes the total outage probability. In the considered problem, the partitioning is made only of singleton and bipartite coalitions:

$$
\min _{\mathcal{M}} \sum_{S \in \mathcal{M}} \sum_{i \in S} p_{\mathcal{O}}^{i}
$$

In the computational results, we find the optimal solution with a brute-force search which enumerates all possible solutions and chooses the one which produces the lowest total outage probability.

\section{Computational Results}

This section includes the comparison of partner selection for different paradigms: stable matching, global optimum, and random selection. In case of random selection, the matching of mobiles is performed randomly. The locations of mobiles are denoted as $\Phi=\left(x_{i}, y_{i}\right)_{i \in N}$ and the destination $\left(x_{d}, y_{d}\right)$ such that the distance between node $i$ and $j$ is given by $d_{i, j}=$ $\sqrt{\left(x_{i}-x_{j}\right)^{2}+\left(y_{i}-y_{j}\right)^{2}}$. Also, we assume that $x_{i}$ and $y_{i}$ follow uniform distribution on some area.
We generate randomly a rate $R$ according to uniform distribution for each mobile; the shadowing component $S$ follows log-normal distribution. In the calculations, the results are obtained per mobile. We utilize the law of total probability which is formulated as $\bar{p}_{\mathcal{O}}(\Theta)=\mathbf{E}_{R, S, \Phi}[\operatorname{Pr}[\mathcal{O} \mid R, S, \Phi]]$, where $\bar{p}_{\mathcal{O}}(\Theta)$ is called as "average outage probability" for any case $\Theta=$ \{Matching, Global Optimum, Random Selection which can be calculated as the ergodic mean over $(R, S, \Phi)$, i.e. $\bar{p}_{\mathcal{O}}(\Theta)=\frac{1}{T} \sum_{t=1}^{T} \operatorname{Pr}[\mathcal{O} \mid R, S$, $\Phi]$, where $T$ is the number of iterations, $R_{t}$ is the rate, $S_{t}$ is the shadowing component, and $\Phi_{t}$ denotes the locations of mobiles in iteration $t$.

\section{A. Test-bed}

In all simulations, we consider the block fading channels with Rayleigh distribution. The variance of the fading is assumed to be 1 . The shadowing variance $\sigma_{S}=8 \mathrm{~dB}$ for all links, the path loss exponent $\beta=3$. The locations $\Phi=\left(x_{i}, y_{i}\right)_{i \in N}$ of mobiles follow uniform distribution within $x \in[85,100], y \in[85,100]$ like a bagel where the location of destination is chosen as $\left(x_{d}, y_{d}\right)=(50,50)$ which could be seen as the center of the bagel. Moreover, additive white Gaussian noise channel is considered in all simulations.

\section{B. Comments and Corollaries}

Figure 2 - Average outage probability with respect to average received SNR: The rate of the mobiles is assumed to be uniformly distributed within $R \in[1,2]$. Also, the number of mobiles is fixed to $n=6$. First, we observe that the cooperation is beneficial to the mobiles for the considered conditions. Note that the result obtained by stable-matching is near to global optimum, and it is better than random selection; for example, there is $3.25 \mathrm{~dB}$ gain when average outage probability is equal to $10^{-4}$.

Figure 3 - Average outage probability with respect to the number of mobiles: Average received SNR is equal to $30 \mathrm{~dB}$, and the distribution of the rate is chosen as $R=[1,3]$. This figure shows that the cooperation is always beneficial on average. Increasing number of mobiles has a positive effect since the probability of finding a good partner increases. Actually, increasing the number of mobiles in some area corresponds with the increasing the intensity of mobiles homogeneously. Observe that there exists a critical value of $n$ that can be seen as a saturation after which the average outage probability becomes constant. For different scenarios, the saturation point changes. We observe here the fact that random selection is not useful compared to the stable-matching result. For example, when $n=10$, the average outage probability is equal to $6.50 \times 10^{-3}$ and $4.36 \times 10^{-3}$ in case of random selection and stable-matching, respectively.

Figure 4 - Probability of cooperation with respect to the number of mobiles: Here, we depict the probability of cooperation of a mobile with another one. The transmitted SNR is $\frac{P}{N_{0}}=(70,75,80,85,90) \mathrm{dB}$. We set the rate to be distributed within $R=[1,3]$. Observe that with increasing transmitted SNR, the probability of cooperation is getting one. However, it is not so while the number of mobiles decreases. This is 
due to the fact that the probability of finding good partner is low when the intensity of mobiles decreases on some area.

Remark 5.1: As a concluding remark about the usage of stable matching algorithm as a partner selection method, we can state that the results related to average outage probability and probability of cooperation show the advantage of Irving's algorithm. It is also fair in terms of the dynamics of matching games where there does not exist a pair that would deviate. Therefore, the decentralized version of this algorithm introduced in Section III-E is very practical for real implementations.

\section{CONCLUSION}

In this paper, we formalized the partner selection problem in decode-and-forward relaying favoured to stable roommates problem. The outage probability for a special protocol has been calculated and chosen as the ranking strategy in the preference lists of players. We proposed a decentralized version of Irving's algorithm for partner selection. Further, we compared the coupling of players with global optimum. In computational results, we showed that stable-matching gives near global optimum results. We also depicted the superior advantage of the stable-matching compared to random selection.

\section{ACKNOWLEDGEMENT}

We would like to thank to Melda Yüksel for her precious comments and recommendations.

\section{REFERENCES}

[1] J. N. Laneman, D. N. C. Tse, and G. W. Wornell. "Cooperative diversity in wireless networks: Efficient protocols and outage behavior", IEEE Trans. on Inform. Theory, vol. 50, no. 12, pp. 3062-3080, Dec. 2004.

[2] D. Gale and L. S. Shapley, "College admissions and the stability of marriage," American Mathematical Monthly vol. 69, pp. 9-14, 1962.

[3] R.W. Irving, "An efficient algorithm for the stable roommates problem," Journal of Algorithms, vol. 6, no. 4, pp. 577-595, 1985.

[4] http://en.wikipedia.org/wiki/Stable_roommates_problem.

[5] Y.S. Jung and Jae Hong Lee, "Partner assignment algorithm for cooperative diversity in mobile communication systems," IEEE VTC 2006-Spring, vol. 4, pp. 1610-1614, 7-10 May 2006.

[6] A. Nosratinia and T. E. Hunter, "Grouping and partner selection in cooperative wireless networks," IEEE J. Sel. Areas Commun., vol. 25, no. 2, pp. 369-378, Feb. 2007

[7] J. Lee; J.H. Lee, "Relay assignment schemes for multiuser DF-AF cooperative wireless networks," IEEE VTC 2011-Fall, pp. 1-5, 5-8 Sept. 2011

[8] M. M. Fareed and M. Uysal, "On relay selection for decode-and-forward relaying," IEEE Trans. Wireless Commun., vol. 8, no. 7, pp. 3341-3346, July 2009.

[9] M. Veluppillai, "Cooperative diversity and partner selection in wireless networks," PhD thesis, Waterloo, Canada, 2007.

[10] M. Abouelseoud and A. Nosratinia, "Heterogeneous relay selection," IEEE Trans. on Wireless Commun., no. 99, pp. 1-9, 2013.

[11] S. Mathur, L. Sankar, and N.B. Mandayam, "Coalitions in cooperative wireless networks," IEEE J. Sel. Areas Commun., vol. 26, no. 7, pp. 1104-1115, Sept. 2008.

[12] A. Mukherjee, H.M. Kwon, "A coalition game framework for decodeand-forward relay networks," IEEE VTC 2009-Fall, pp.1-5, 20-23 Sept. 2009.

[13] D. Niyato, P. Wang, and W. Saad, Z. Han; A. Hjorungnes, "Coalition formation games for relay transmission: Stability analysis under uncertainty," IEEE WCNC 2011, pp. 920-925, 28-31 March 2011.
[14] P. Biró, "The stable matching problem and its generalizations: an algorithmic and game theoretical approach," PhD Thesis, Budapest, Sept. 2007.

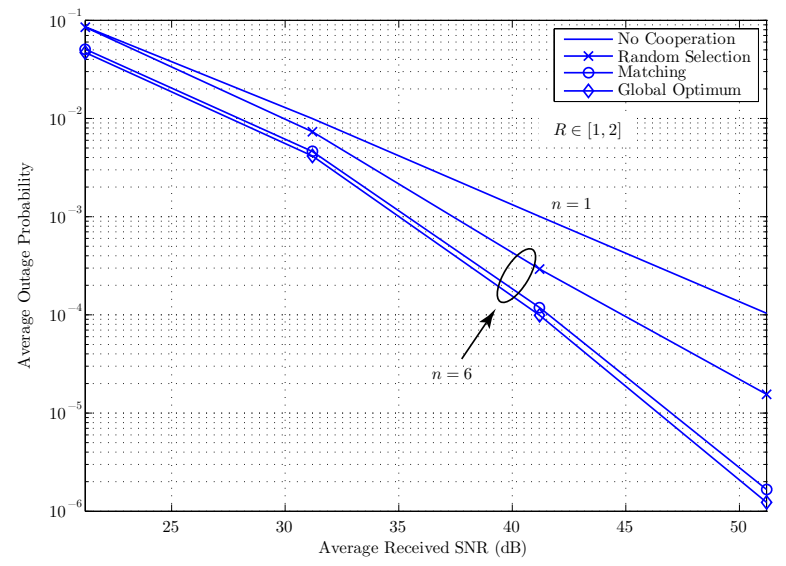

Fig. 2. Average outage probability with respect to average received SNR.

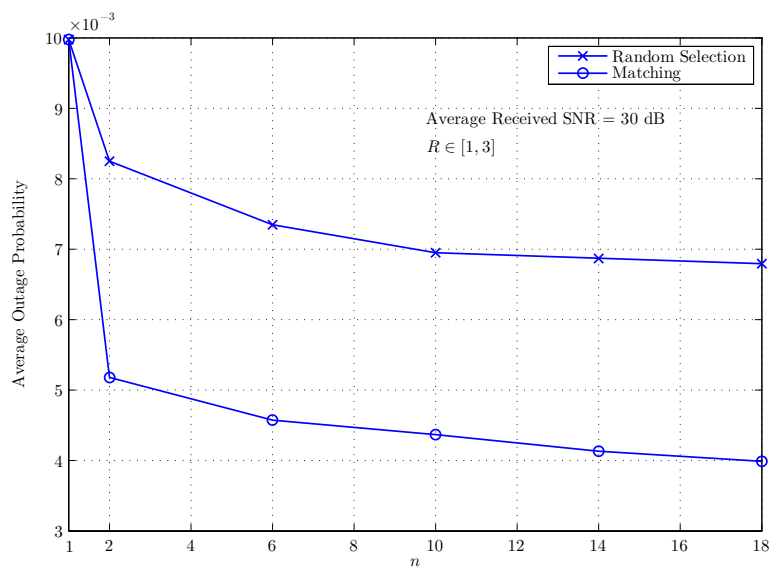

Fig. 3. Average outage probability with respect to the number of mobiles.

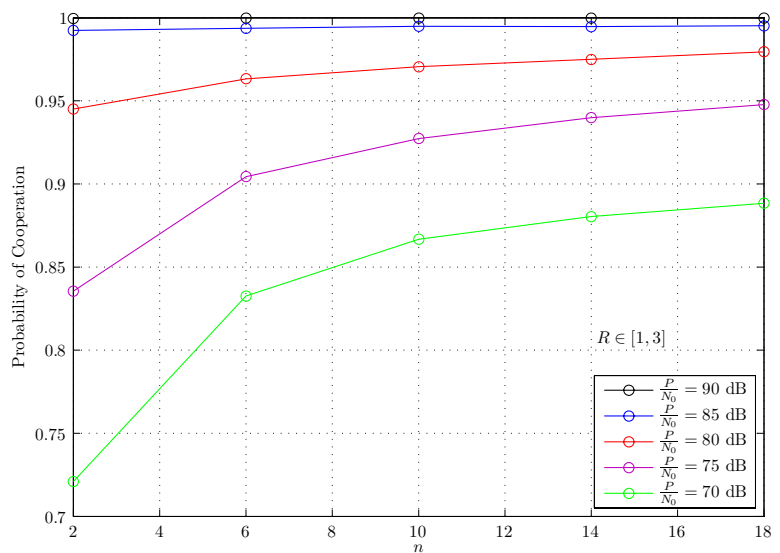

Fig. 4. Probability of cooperation with respect to the number of mobiles. 